\title{
PEDIATRIC EDITORIAL Deprivation indices and childhood BMI: a re-evaluation
}

\author{
International Journal of Obesity (2013) 37, 475-476; \\ doi:10.1038/ijo.2013.3; published online 12 February 2013
}

A paper in this issue ${ }^{1}$ challenges the conventional wisdom that body mass index (BMI) in children increases as deprivation index score increases. These results should lead us to consider both our assumptions about the data, but also our assumptions about the 'shape' of health inequalities.

Deprivation indices were developed to identify geographic areas within the UK, which have multiple economic, social and housing disadvantage. The UK measures are among the most sophisticated in the world and are widely used in policy and planning across multiple departments of the UK government ${ }^{2}$ as well as outside of government. A large body of research has demonstrated that mortality, life expectancy and many health problems are associated in a graded fashion with deprivation score such that health, however defined, worsens as deprivation increases. The causes of ill-health/mortality are also graded across deprivation score and it has been widely reported that (1) there is an increasingly greater prevalence of health-adverse behaviours with increasing deprivation and, (2) that healthadverse behaviours cluster together. ${ }^{3}$ The latter observation is somewhat confounding, as there is nothing inherent about being poor or middle class that would 'force' a person to smoke or refuse to exercise. And, here it is important to note another interesting point; in spite of the term 'deprivation' this graded relationship between deprivation and ill-health/mortality, etc. is indeed graded even among the less deprived!

This previous body of research means that any negative aspect of health is presumed to increase in prevalence as deprivation score increases. In this context the current body of $\mathrm{BMI}$ research is almost completely focussed on overweight and obesity, and the assumptions made about the relationship between BMI and deprivation are of a graded relationship of increasingly greater prevalence of overweight/obesity, with increasing deprivation index score, with the purported mechanisms being overeating and lack of exercise (see page 33 ). ${ }^{4}$ The problem with this assumption is that both the extremes of $\mathrm{BMI}$ are unhealthy, and therefore BMI has a u-shaped association with mortality and morbidity ${ }_{i}^{5,6}$ this relationship is well-established and has been maintained across the 20th century. ${ }^{7}$

Historically, malnutrition, usually diagnosed with an 'underweight' BMI, was a significant health issue but because of the steady increase in mean population BMI over the previous few decades, there is an assumption that underweight is not really a health problem anymore except for small numbers of people with body image disorders. Griffiths' paper clearly shows that this assumption, at least for children may be wrong. Considerable previous research has reported a statistically significant positive association between overweight and obesity and deprivation score using linear regression techniques. But the analysis reported here found that mean BMI peaked in middle-class deprivation scores. We do not actually know whether other researchers have not seen this curvilinear relationship between BMI and deprivation or that they have not tested for its presence because they assumed a linear relationship.

It may also be that the previously reported positive association between BMI and deprivation only 'appeared' as the proportion of underweight people in the population has declined. This would lead to a more j-shaped joint association, which is sufficiently linear to result in a statistically significant association. Another possible explanation for the lower mean BMI in this study's more deprived groups is that there are more children who are undernourished, which would have the effect of reducing the mean level of the BMI in the most deprived. An examination of the variance in BMI levels by deprivation level or a plot of the frequency distribution at each level of deprivation would demonstrate whether this latter explanation is the case.

Regardless of the underlying reasons for the nonlinear relationship it is crucial that health researchers become more aware of the possibility of nonlinear relationships, as they are actually quite common in biology. ${ }^{8}$ Within public health this is particularly noticeable in the area of health-adverse behaviours. The most overhyped of which is probably the nonlinear relationship between cardiovascular disease and alcohol consumption, ${ }^{9}$ such that abstainers and people with high consumptions levels are at the most risk of developing cardiovascular disease. A similar relationship is seen with exercise and cardiovascular disease. ${ }^{10}$

Researchers need to stop assuming that the relationships are linear and start practicing what most of us were taught in introductory statistics - to do some exploratory data analysis at the beginning of the analysis. Unfortunately taking this too far has been perceived as 'fishing expeditions', and we are not encouraged to devote the time needed to understand our data. The fundamental questions we need to ask before we dive into the 'interesting' part of the analysis are: (1) What is the nature of the relationship between variables? (2) Should linear relationships be assumed? (3) Do the data actually conform to the underlying statistical assumptions for the analytic method selected?

It is essential for effectively targeting interventions that we have a genuine understanding of how the risk factor is distributed in the population. We do not necessarily have to try to use complex analytic techniques, which I have found to be difficult to explain clearly in a journal article. But when we provide data plots, as in the Griffiths paper, this will clearly make the point much more effectively than complicated mathematical transformations in regression tables.

The overall message is to spend quality time with the data and check all assumptions.

\section{CONFLICT OF INTEREST}

The authors declare no conflict of interest.

SJ Kelly

Reader in Public Health, Centre for Health and Social Care Research, Sheffield Hallam University, Sheffield, UK

E-mail: S.Kelly@shu.ac.uk

\section{REFERENCES}

1 Griffiths C, Gately P, Marchant PR, Cooke CB. Area-level deprivation and adiposity in children: is the relationship linear? Int J Obesity 2013; 37: 486-492.

2 UK Office for National Statistics. UK indices of multiple deprivation - a way to make comparisons across constituent countries easier 2012.

3 Shankar A, McMunn A, Steptoe A. Health-related behaviors in older adults: Relationships with socioeconomic status. Am J Prev Med 2010; 38: 39-46.

4 Marmot M. Fair Society, Healthy Lives: The Marmot Review. WHO, 2010. 
5 Flegal KM, Graubard Bl, Williamson DF, Gail MH. Cause-specific excess deaths associated with underweight, overweight, and obesity. JAMA 2007; 298: 2028-2037.

6 Kivimaki M, Ferrie JE, Batty GD, Davey SG, Elovainio M, Marmot MG et al. Optimal form of operationalizing BMI in relation to all-cause and cause-specific mortality: the original Whitehall study. Obesity 2008; 16: 1926-1932.

7 Linares C, Su D. Body mass index and health among Union Army veterans: 1891-1905. Econ Hum Biol 2005; 3: 367-387.
8 Calabrese EJ, Baldwin LA. U-shaped dose-response in biology, toxicology, and public health. Annu Rev Public Health 2001; 22: 15-33.

9 Burger M, Mensink G, Bronstrup A, Thierfelder W, Pietrzik K. Alcohol consumption and its relation to cardiovascular risk factors in Germany. Eur J Clin Nutr 2004; 58: 605-614.

10 O'Keefe JH, Patil HR, Lavie CJ, Magalski A, Vogel RA, McCullough PA. Potential adverse cardiovascular effects from excessive endurance exercise. Mayo Clin Proc 2012; 87: 587-595. 